\title{
IL-10 modulates depressive-like behavior
}

\section{Ana Raquel Mesquita a , Margarida Correia-Neves ${ }^{\mathrm{a}}$, Susana Roque ${ }^{\mathrm{a}}$, António Gil Castro ${ }^{\mathrm{a}, \mathrm{b}}$, Paulo Vieira ${ }^{\mathrm{b}, 1}$, Jorge Pedrosa ${ }^{\mathrm{a}}$, Joana Almeida Palha ${ }^{\mathrm{a}}$, Nuno Sousa ${ }^{\mathrm{a}, *}$ \\ ${ }^{a}$ Life and Health Sciences Research Institute (ICVS), School of Health Sciences, University of Minho, Campus de Gualtar, $4710-057$ Braga, Portugal b Instituto Gulbenkian de Ciência, 2781 Oeiras, Portugal}

\section{Abstract}

The role of pro-inflammatory cytokines in psychiatric disorders has been the focus of great research attention in recent years. Paradoxically, the same is not true for anti-inflammatory cytokines. In the present study, we assessed the behavioral profile of animals with altered expression of the anti-inflammatory cytokine IL-10.

We performed a battery of tests to assess anxiety, depressive-like and cognitive behaviors in mice overexpressing IL-10 (PMT10) and IL- $10^{-I-}$ animals; in the later mice we also tested the behavioral effect of IL-10 administration.

In the forced-swimming test, IL-10 $0^{-1-}$ females displayed increased depressive-like behavior; importantly, this phenotype was reverted by the injection of IL-10. Moreover, mice overexpressing IL-10 presented a decreased depressive-like behavior. Despite the presence of a similar trend, male animals did not reach significant differences in depressive-like behavior. Assessment in the open-field showed that the absence of IL-10 decreased the percentage of time spent in the center of the arena in both male and female mice, while male animals overexpressing IL-10 revealed an opposite behavior. For both sexes, imbalance in IL-10 levels did not affect spatial reference memory.

In conclusion, variations in IL-10 expression are associated with an altered depressive-like behavior, but do not influence cognitive performance. Interestingly, IL-10 imbalance produced more profound behavioral changes in females than in male animals. This is in accordance with clinical data demonstrating an increased susceptibility of women to mood disorders, suggesting an interplay between anti-inflammatory cytokines and sexual steroids.

(C) 2008 Published by Elsevier Ltd.

Keywords: Anti-inflammatory cytokines; Depression; Anxiety; Cognition; Sexual steroids

\section{Introduction}

The cross-talk between the central nervous system (CNS) and the peripheral immune system has been a subject of great research interest in recent years. Since the discovery of cytokine receptors in both glial cells and neurons (Araujo et al., 1989; Breder et al., 1988; Cunningham and De Souza, 1993; McGeer and McGeer, 1995). The influence of cytokines in brain function, resulting either from

\footnotetext{
* Corresponding author. Tel.: +351 253604806; fax: +351 253604809. E-mail address: njcsousa@ecsaude.uminho.pt (N. Sousa).

${ }^{1}$ Present address: Institut National de la Santé et de la Recherche Médicale (INSERM) U 668, and Unité du Développement des Lymphocytes, Institut Pasteur, Paris, France.
}

signaling by cytokines produced peripherally or within the CNS (mainly by microglia and astrocytes) (Araujo et al., 1989; Breder et al., 1988; Cunningham and De Souza, 1993; McGeer and McGeer, 1995), has been characterized. Several studies have highlighted the role of cytokines in neuropathogenesis, particularly in mood disorders (Papanicolaou et al., 1998; Schiepers et al., 2005; Yirmiya et al., 2000). Indeed, one of the most consistent observations in the field is the increased levels of pro-inflammatory cytokines in depression (Lanquillon et al., 2000; Maes et al., 1995b; Mikova et al., 2001; Sluzewska et al., 1995; Tuglu et al., 2003).

In an attempt to integrate the above described findings, it has been proposed that the release of pro-inflammatory cytokines (mostly IL- $1 \beta$, IL-6, IFN- $\gamma, \mathrm{TNF}-\alpha$ ) is a major 
determinant for the behavioral, neuroendocrine and neurochemistry alterations associated with depressive disorders (Maes et al., 1993). Whether these changes in cytokine expression are the cause or consequence of depression is still a matter of dispute, but the demonstration that the immune dysregulation precedes the development of depression (Yirmiya et al., 2000) is of notice. In support of the role of cytokines in mood disorders are the observations of early depressive symptoms in patients receiving interferon and IL-2 therapy (Capuron et al., 2000); interestingly, subsequent studies have also shown that these depressive symptoms can be relieved by the administration of antidepressants (Musselman et al., 2001; Yirmiya et al., 2001). The role of cytokines in mood disorders is further strengthened by the demonstration that pro-inflammatory cytokines are able to activate the hypothalamus-pituitary-adrenal (HPA) axis (Crane et al., 2003). The overactivation of the HPA is one of the most recognized findings in mood disorders and considered to be an important trigger of these psychopathologies (Carroll et al., 1968; Plotsky et al., 1998). Moreover, increases in both IL-1 $\beta$ (Linthorst et al., 1995; Merali et al., 1997; Shintani et al., 1993; Song et al., 1999) and TNF (Brebner et al., 2000; Hayley et al., 1999) have been associated with alterations at the neurochemical level, predominantly in the serotoninergic system (Dunn et al., 1999), in several brain regions known to be implicated in depression. As a consequence, animals exposed to increase levels of pro-inflammatory cytokines display signs of depressive-like behavior (De La Garza, 2005; Dunn et al., 2005) and increased anxiety (Silverman et al., 2007).

While several reports implicate pro-inflammatory cytokines in behavior, less is known on the influence of antiinflammatory cytokines. Among the few studies on the subject, administration of IL-10 prior to LPS injection has been shown to revert the behavioral effects of LPS injection (Bluthe et al., 1999), including the effects upon mobility, rearing activity and social exploration and interaction (Leon et al., 1999; Nava et al., 1997; Smith et al., 1999). Several authors have hypothesized that the behavioral effects of IL-10 are a consequence of an inhibitory effect on IL-1, INF- $\gamma$ and TNF production and not a direct effect of this anti-inflammatory molecule; in fact, it is known that IL-10 is important on the down-modulation of these proinflammatory cytokines (Fiorentino et al., 1991; Harvey et al., 2006; Moore et al., 2001). However, more recently, it was demonstrated that IL-10 administration to animals without exposure to inflammatory challenge induces increased motor activity and abnormal exploratory patterns (Harvey et al., 2006), which indicates that this cytokine might directly influence behavior. In this study, we aimed to further investigate whether manipulation of IL10 levels could modulate behavior. To achieve this goal, we examined the behavioral profile of IL-10 $0^{-/-}$and transgenic mice that overexpress this anti-inflammatory cytokine (PMT10); moreover, to further assess the influence of this cytokine in modulating behavior, we analyzed the effects of IL-10 administration to IL- $10^{-/-}$mice.

\subsection{Animals}

The IL-10 $0^{-1-}$ animals on a Balb/c background were bred in our animals facilities from a breeding pair provided from Dr. A. O'Garra (National Institute for Medical Research, London, UK). For behavioral characterization of these knock-out animals, we used $40 \mathrm{IL}-10^{-/-}(23$ females and 17 males) on a Balb/c background and 31 wild-type Balb/c (17 females and 14 males) as respective controls. All animals were genotyped by PCR. As some IL-10 ${ }^{-/-}$animals develop spontaneous inflammatory bowel disease, analysis of bowels was carefully performed throughout the experimental period and diarrheic animals were excluded from this study.

PMT10 animals on a C57BL/6 background were produced by Drs. P Vieira and AG Castro. A mouse IL-10 cDNA sequence was cloned in the p169ZT vector (Sousa et al., 2002), which carries the sheep metallothioneinc (MT) Ia promoter (Peterson and Mercer, 1986), a $\beta$-globin splice site and the SV40 polyadenylation signal. The resulting vector (pMT-IL10) was injected in C57BL/6 eggs and transgenic founders were identified by PCR using MT-specific primers. The presence of the transgene was confirmed by Southern blot analysis encompassing the sheep MT-promoter. PMT10 mice were bred at our animal facilities. In this experiment, ten PMT10 animals and ten wild-type C57BL/6 as respective controls were used. IL-10 overexpression was induced by giving a $2 \%$ sucrose solution with $50 \mathrm{mM}$ of zinc sulfate to animals ad libitum. As the IL-10 promoter is associated with a metalloprotein, the presence of zinc in this solution induces its activation. Wild type animals were also supplied with the same drinking solution.

Serum levels of IL-10, which range between 3 and $5 \mathrm{ng} /$ $\mathrm{ml}$, could be measured 3 days after induction and remained stable while the animals were drinking the zinc solution. IL-10 was never detected in the serum of non-transgenic littermates or in non-induced transgenic mice. IL-10 overexpression was induced 1 week before the behavioral testes were initiated.

All mice were kept in an animal facility in a $12 \mathrm{~h}$ light: $12 \mathrm{~h}$ dark cycle, with food and water available ad libitum. Males and females were kept separately. At 3 months of age all animals were behaviorally tested between 10 a.m. and 5 p.m. in a counterbalanced order; IL- $10^{-/-}$mice were compared to wild-type Balb/c, whereas PMT10 animals were compared to wild-type C57BL/6 mice. At the end of the experiment, animals were sacrificed by decapitation; decapitation was performed by trained operators. All experimental procedures were conducted in accordance to the European Communities Council Directive, 86/609/EEC.

\subsection{IL-10 injection}

Another subset of 20 females and 16 males of IL-10 $0^{-/-}$ mice were used in a supplementary experiment in which 
they were injected with recombinant mouse IL-10 (R\&D systems, Minneapolis, USA). Half of these animals received a daily i.p. injection of $40 \mathrm{ng}$ of IL-10 for 11 days ( 6 days prior the behavioral analysis followed by 5 more days concomitant with the behavioral assessment), while control animals were injected with a saline solution.

\subsection{Cytokine measurements}

Serum levels of INF- $\gamma$ were measured by a two-side sandwich ELISA with the anti-IFN- $\gamma$ specific affinity-purified mAbs (R4-6A2 as capture and biotinylated AN-18 as detecting $\mathrm{mAbs}$ ), and a standard curve was generated with known amounts of IFN- $\gamma$ (Peprotech, Rocky Hill, NJ, USA). The sensitivity of the assay was $16 \mathrm{pg} / \mathrm{ml}$.

Quantification of TNF- $\alpha$ was done by ELISA (Duo Set; R\&D Systems, Minneapolis, USA). The sensitivity of the assay was $32 \mathrm{pg} / \mathrm{ml}$.

Serum levels of IL-10 were determined by ELISA (Quantikine; R\&D Systems, Minneapolis, USA). The sensitivity of the assay was $4 \mathrm{pg} / \mathrm{ml}$.

\subsection{Behavioral analysis}

\subsubsection{Open-field test}

Animals were placed in the center of the arena $(43.2 \times 43.2 \mathrm{~cm}$ transparent acrylic walls and white floor) and their position was monitored and recorded by a two 16-beam infrared system (MedAssociates, Vermont, USA), over a period of $5 \mathrm{~min}$. Time in the predefined central and peripheral areas was recorded and used to evaluate anxious-like behavior, while the total distance traveled assessed spontaneous activity. The number and duration of the "rearings" (vertical activity) were also recorded as a measure of exploratory behavior.

\subsubsection{Elevated-plus maze}

Animals were placed in a elevated-plus maze (EPM) apparatus consisting of two opposite open arms $(50.8 \times 10.2 \mathrm{~cm})$ and two opposite closed arms $(50.8 \times 10.2 \times 40.6 \mathrm{~cm})$ raised $72.4 \mathrm{~cm}$ above the floor (ENV-560, MedAssociates, Vermont, USA). The number of entries and the time spent in each arm was registered by an infrared system over a total test period of $5 \mathrm{~min}$.

\subsubsection{Forced-swimming test}

Animals were placed in a cylinder (diameter: $37 \mathrm{~cm}$; $55 \mathrm{~cm}$ of height $)$ filled with water $\left(25^{\circ} \mathrm{C}\right)$ to $35 \mathrm{~cm}$ depth such as that they had no solid support for the rear paws nor for the tail. Animals were tested in a $5 \mathrm{~min}$ session $24 \mathrm{~h}$ after being exposed to the test for the same time period. Only the second session was recorded and later scored by two independent researchers which were blind to the experimental conditions. Time of immobility and latency to immobility were computed and used as a measure of depressive-like behavior.

\subsubsection{Morris water maze}

This maze consisted of a tank (diameter: $170 \mathrm{~cm}$; depth: $50 \mathrm{~cm}$ ), divided into quadrants by imaginary lines and filled with opaque water to a depth of $31 \mathrm{~cm}$. During testing, a platform $(12 \times 12 \mathrm{~cm}$; invisible to the mice $)$ was placed in the same quadrant during five consecutive days. Each test session consisted of four trials which lasted in maximum for $2 \mathrm{~min}$. Time spent swimming to reach the hidden platform was recorded and used to evaluate learning and memory performances.

\subsection{Statistical analysis}

All the results from the behavioral tests are expressed as mean \pm standard error of the mean (SEM). Statistical analyses were performed using SPSS 15.0 software (SPSS Inc., Chicago/IL). The effect of gender and IL-10 expression, per se, in the Open Field and the forced swimming tests were analyzed by independent samples $t$-test, while the overall effects were studied by two-way analysis of variance (ANOVA).

Data from the Morris water maze task was analyzed using a repeated measures ANOVA analysis throughout the 5 days test. Each day is a mean of the four consecutive trials. All behavioral results are expressed as means \pm SE and statistical significance was considered for $p$ values $\leqslant 0.05$.

\section{Results}

\subsection{Biometric parameters}

Analysis of the relative weight of thymus and adrenal glands from IL- $10^{-/-}$animals compared with wild-type animals showed a significant reduction of the thymus in both male and female mice $(t=3.9 ; p \leqslant 0.001$ and $t=2.9 ; p<0.05$, respectively) (Fig. 1A and B). Two-way
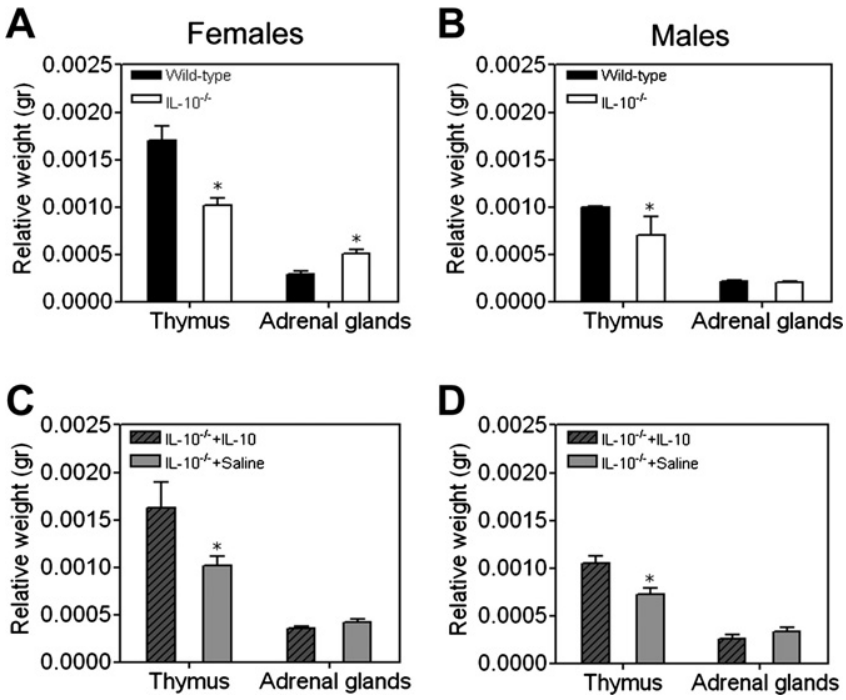

Fig. 1. Thymus and adrenal glands weight relative to the body weight of Wild-type and $\mathrm{IL} 10^{-/-}$females and males (A and B) and IL-10 ${ }^{-/-}$ receiving IL-10 (IL-10 ${ }^{-/-}+$IL-10) and IL- $10^{-/-}$receiving saline (IL-10 ${ }^{-/}$ - Saline) animals (C and D). Values are means $\pm \mathrm{SE}$ and ${ }^{*} p<0.05$. 
ANOVA revealed a gender effect on adrenals weight $\left(F_{1,27}=24.1 ; p<0.001\right)$, with interaction between gender and IL-10 factors. Interestingly, IL-10 administration reverted the atrophy of thymus in $\mathrm{IL}-10^{-/-}$mice $(t=-3.1 ; p<0.05$ for females and $t=-3.5 ; p<0.05$ for males) (Fig. 1C and D).

Regarding the adrenals weight, a significant increase was found in females IL-10 $0^{-1-}(t=-4.5 ; p<0.001)$ (Fig. 1A). Once more, IL-10 administration showed to be effective in restoring the adrenals weight in $\mathrm{IL}-10^{-/-}$females $(t=2.5 ; p<0.05$; Fig. 1C). No statistical significance was found in males (Fig. 1B and D). Two-way ANOVA showed a gender effect on both biometric parameters $\left(F_{1,31}=13.8 ; \quad p<0.05\right.$ for thymus and $F_{1,31}=9.3$; $p<0.05$ for adrenal glands).

\subsection{IL-10 production influences depressive-like behavior in female mice}

In the forced-swimming test (FST) we evaluated the ability of mice to cope with a stressful and inescapable situation (learned helplessness). In this test, animals displaying decreased latency to immobility and longer immobilization periods are considered to have increased helplessness, which is a sign of depressive-like behavior. When tested in the FST, IL- $10^{-1-}$ female showed decreased activity $(t=6.1$, $p<0.001)$ during the $5 \mathrm{~min}$ of the test and stopped swimming earlier than wild-type mice $(t=3.3 \quad p<0.005$ (Fig. 2A). No significant differences were observed between male animals (Fig. 2B). Two-way ANOVA revealed a significant effect of gender, being the females more affected than males in latency to immobility $\left(F_{1,67}=14.0\right.$, $p \leqslant 0.001)$ and activity times $\left(F_{1,67}=5.2, p \leqslant 0.05\right)$.

Interestingly, IL-10 administration was able to reverse the depressive-like phenotype of IL- $10^{-/}$females, in as much as after administration of this cytokine there was an increased activity time namely when compared with animals receiving saline injection $(t=-2.9, p<0.05)$ (Fig. 2C). Although this difference was only observed in females, a similar trend was also found in male mice (Fig. 2D).

Further supporting the anti-depressant action of IL-10, PMT10 female mice showed increased activity time when compared with their respective counterparts $(t=-5.5$; $p \leqslant 0.001$ ), which reveals a decreased susceptibility to depressive-like behavior (Fig. 2E). Although male animals showed a similar behavioral pattern, no statistical significant differences were observed in any of the parameters analyzed (Fig. 2F). Again, two-way ANOVA showed a significant effect of gender in the activity time $\left(F_{1,16}=6.5\right.$, $p \leqslant 0.05$ ), being the females more vulnerable to IL-10 imbalances.

\subsection{Variations in IL-10 levels affect anxious-like behavior in the open-field}

The open-field test (OF) was performed to assess general locomotor activity and exploratory behavior. In terms of
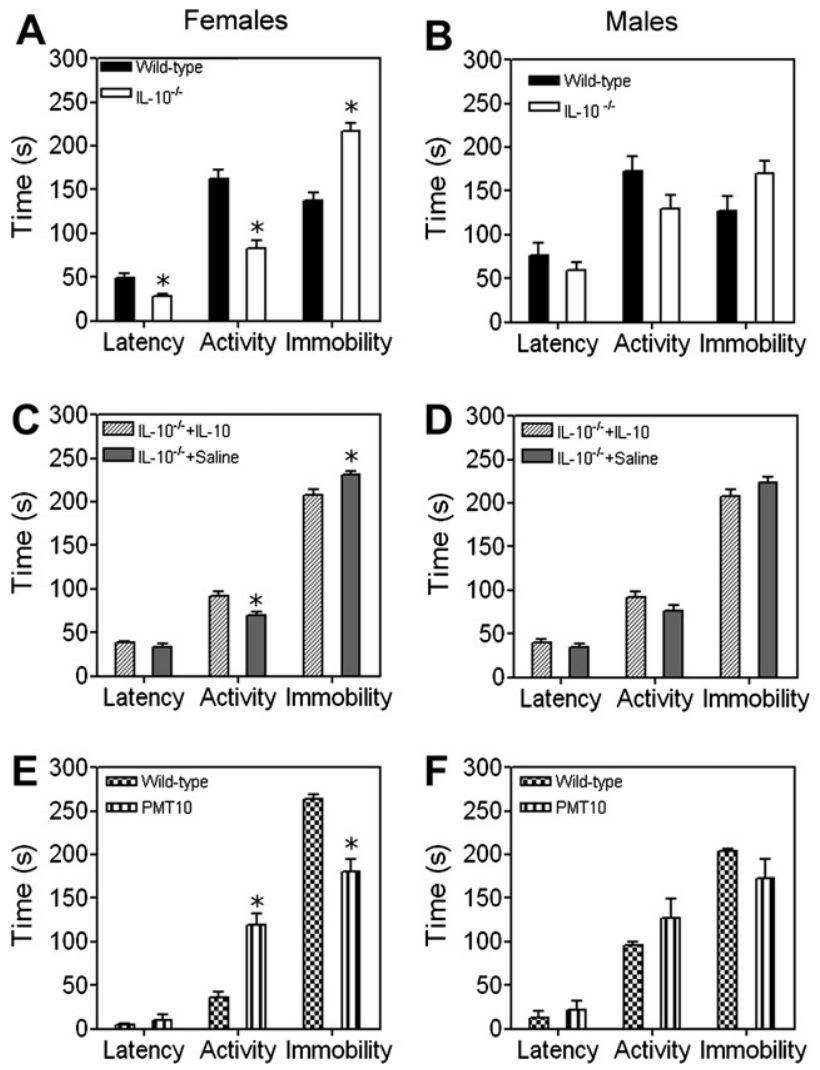

Fig. 2. Depressive-like behavior assessed with the forced-swimming test (FST). IL- $10^{-1-}$ females displayed increased immobility time and decrease latency to immobility compared with their wild-type controls (A). No significant effect was observed in male mice (B). Administration of IL-10 was only able to increase activity time in female IL- $10^{-1-}$ mice, when compared with $\mathrm{IL}-10^{-/-}$mice receiving saline injection $(\mathrm{C}$ and $\mathrm{D})$. Female animals overexpressing IL-10 (PMT10) revealed an increase activity time in the FST (E). The same tendency was obtained for male PMT10 animals (F). Values are means \pm SE and ${ }^{*} p<0.05$.

spontaneous activity, assessed by the total distance traveled throughout the $5 \mathrm{~min}$ of the test, no differences were observed between IL-10 $10^{-/-}$and wild-type animals. Moreover, both IL-10 administration and overexpression failed to induce any change in this behavior both in males and females.

Analysis of the exploratory behavior in terms of animals' vertical activity (number and duration of rearings) revealed a significant increase in both measurements in IL- $10^{-/-}$female mice compared to their wild-type counterparts (Fig. 3A). No significant differences were observed in male animals, although there was a trend for increased exploratory behavior in IL-10 $10^{-/-}$animals (Fig. 3B). Both the administration and the overexpression of IL-10 failed to show any effect in vertical activity (Fig. 3C, D and E, F), despite a tendency for reduction after IL-10 administration in male IL- $10^{-/-}$mice.

Although the OF is not the most widely used test to assess anxious-like behavior, the percentage of time spent in center of the arena over the total time provides an indicative measure of anxiety-behavior (Boguszewski and Zagrodzka, 2002; Ramos et al., 1997; Simen et al., 2006). 

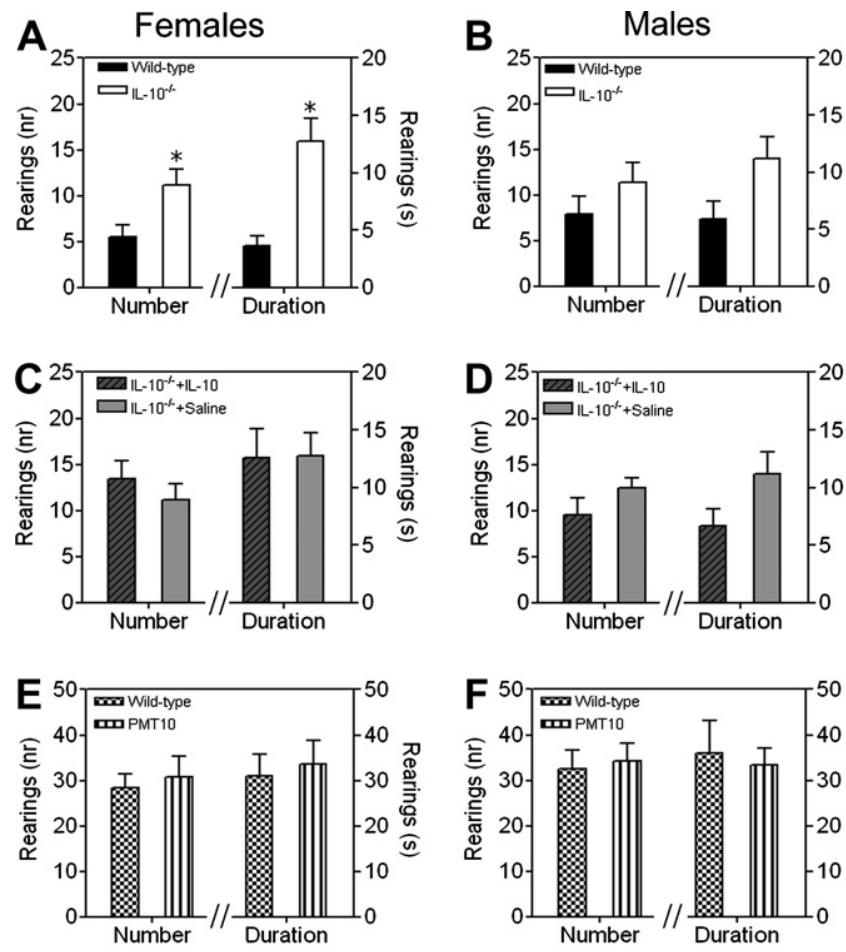

Fig. 3. Vertical activity assessed by the number and duration of rearings in the open-field test (OF), showed increased exploratory behavior in IL$10^{-/-}$female but not in males (A and B); IL-10 administration did not change rearing activity neither in male or female mice $(C$ and $D)$. No significant differences in rearing activity were observed between PMT10 and respective control animals (E and F). Values are means $\pm \mathrm{SE}$ and ${ }^{*} p<0.05$.

Analysis of this parameter demonstrated that both female and male IL- $10^{-/-}$mice spent significantly less time in the center than in the periphery when compared to wildtype animals $(t=2.6, p<0.05, t=4.0, p \leqslant 0.001$, respectively) (Fig. 4A and B).

Interestingly, the IL-10 treatment was able to reverse the IL-10 ${ }^{-/-}$mice phenotype, in males, resulting in increased time spent in the center of the OF arena $(t=-2.9$, $p<0.05)$. Two-way ANOVA revealed a gender effect in this parameter $\left(F_{1,32}=4.6, p<0.05\right)$ (Fig. $4 \mathrm{C}$ and D).

The same trend was observed for PMT10 animals which spent significantly more time in the center of the OF, even though differences were only significant in males $(t=-4.3$, $p \leqslant 0.05$; Fig. 4E and F).

However, care must be taken in the interpretation of these results as no differences were found in the EPM in neither IL- $10^{-/-}$nor in PMT10 mice when compared with respective control animals (data not shown).

\subsection{IL-10 production did not affect hippocampal-dependent spatial memory}

To investigate whether changes in IL-10 "milieu" influence hippocampal-dependent learning and memory, the Morris water maze (MWM) test was performed. No differences were found in both male and females regarding the
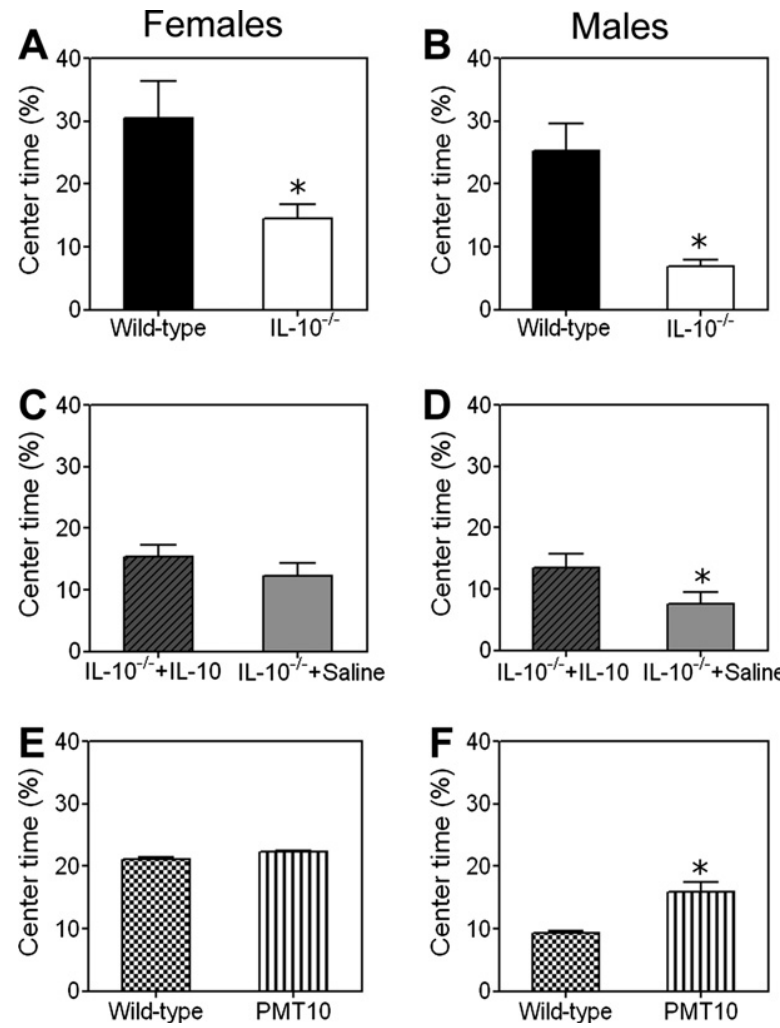

Fig. 4. Anxious-like behavior assessed by the time spent in the center of the OF arena, showed increased anxiety in IL- $10^{-1-}$ (A and B). However, administration of IL-10 only reversed the phenotype in IL-10 ${ }^{-1-}$ male (C and D). Male PMT10 spent more time in the center than control animals a sign of decreased anxious-like behavior (E and F). Values are means of the percentage of time spent in the center over the total time $\pm \mathrm{SE}$ and ${ }^{*} p<0.05$.

time and the distance swam to find the hidden platform (Fig. 5), thus showing that neither the overexpression nor the absence of IL-10 seem to affect spatial memory.

\subsection{Changes in $I L-10$ expression did not induce detectable changes in TNF- $\alpha$ and IFN- $\gamma$ levels}

In order to investigate whether genetic manipulation of IL-10 expression influences the production of two relevant pro-inflammatory cytokines, the serum levels of TNF- $\alpha$ and INF- $\gamma$ were measured. No differences were observed between PMT10 and IL- $10^{-/-}$and their respective wildtype controls as determinations for both cytokines in the serum were below the detection levels $(16 \mathrm{pg} / \mathrm{ml}$ for TNF$\alpha$ and $32 \mathrm{pg} / \mathrm{ml}$ for INF- $\gamma$ ).

\section{Discussion}

By studying emotional behavior in mice lacking or overexpressing IL-10, we herein show that this anti-inflammatory cytokine influences depressive-like behavior. Mice lacking IL-10 displayed signs of depressive-like behavior, assessed by immobilization time and latency to immobility, when compared to their strain-matched wild-type counterparts. In contrast, both PMT10 and $\mathrm{IL}-10^{-/-}$receiving this 
A

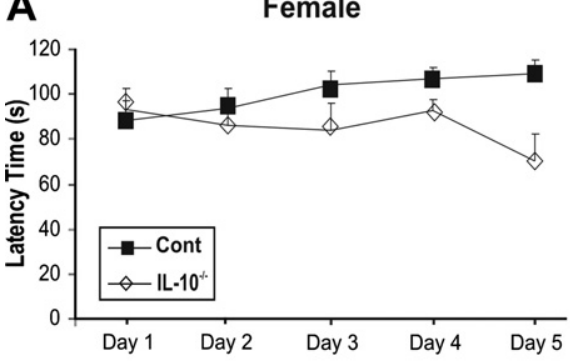

C

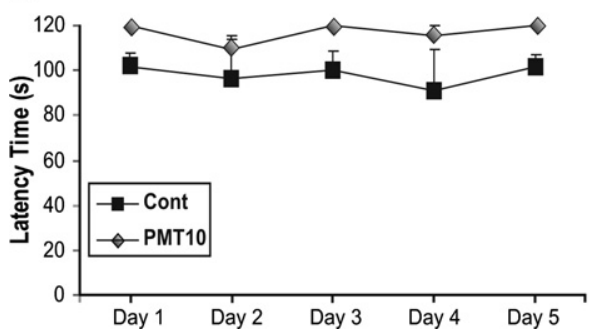

B

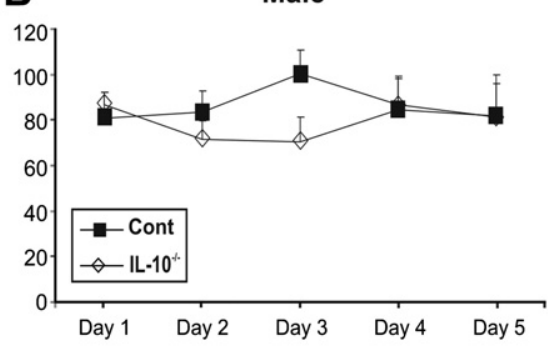

D

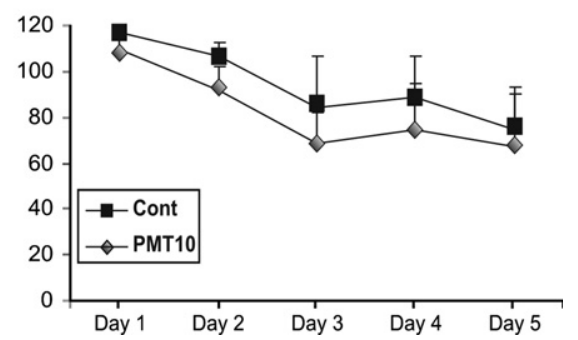

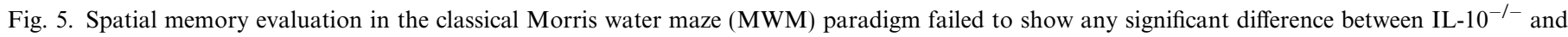

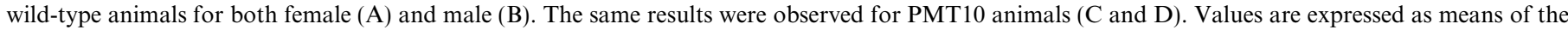
four trials/day $\pm \mathrm{SE}$ and ${ }^{*} p<0.05$.

cytokine displayed an opposite behavioral phenotype. Remarkably, variation in IL-10 levels affected more profoundly females, which correlates with the recognized higher susceptibility of women to depression. Taken together, these results reveal, for the first time, that this anti-inflammatory cytokine is an important mediator in depression.

This observation adds to the so-called "cytokine hypothesis of depression", that was built on the evidence that pro-inflammatory cytokines have a trigger effect on the pathogenesis of depression (Maes et al., 1995a; Maes et al., 1993). This effect was proposed to be mediated by neuroendocrine and neurotransmitter systems involved in vulnerability to affective disorders (Maes, 1999). In this respect it has been demonstrated that IL-1 $\beta$ and TNF- $\alpha$ stimulate the expression/release of corticotrophin-releasing hormone (CRH) in the paraventricular nucleus (PVN) of the hypothalamus (Hayley et al., 2001; Tilders and Schmidt, 1998), the control center of the HPA axis and alters the turnover of norepinephrine and serotonin (5HT) in the hypothalamus, amygdala, prefrontal cortex, and hippocampus (Ando and Dunn, 1999; Brebner et al., 2000; Dunn et al., 1999; Hayley et al., 1999). Further evidence for the role of pro-inflammatory cytokines in depression was gathered from studies with TNF receptors (TNFR) knock-out mice, in which it was shown that both TNFR $1^{-/-}$and TNFR $2^{-/-}$mice were more active in the FST than wild-type animals (Simen et al., 2006). Importantly, the mechanisms underpinning the behavioral changes in these mice models are similar and include alterations in neurotransmission in regions of the brain implicated in emotional behavior. Of notice, is also the evidence of the immunomodulatory effects of antidepres- sants that act preferentially in the noradrenergic and serotoninergic system. Kubera and co-workers (Kubera et al., 2001) demonstrated the ability of different drugs to decrease the levels of INF- $\gamma$, while increasing the levels of the anti-inflammatory cytokine IL-10. These data were also corroborated by studies using other antidepressants in stimulated human blood cells (Maes et al., 1999).

While the involvement of pro-inflammatory cytokines in many aspects of depressive illness is now indisputable, we clearly demonstrate in this study that anti-inflammatory cytokines also influence emotional behavior in rodents. Work from Bluthe and collaborators (Bluthe et al., 1999) had already suggested that IL-10 administration could abrogate the behavioral effects of LPS injection in sickness behavior in rats. It was postulated that IL-10 inhibits the expression of pro-inflammatory cytokines (IL-1, INF- $\gamma$ and $\mathrm{TNF}-\alpha$ ) produced in response to LPS and its behavioral consequences, increasing the duration of social interaction. The present data reveals that variations in IL-10 expression influence mood behavior, even in the absence of detectable variations in the serum levels of INF- $\gamma$ and TNF- $\alpha$. This fact, however, does not exclude the possibility of an inhibition of pro-inflammatory cytokines secretion in the CNS; in accordance, IL-10 deficient mice have increased brain levels of TNF- $\alpha$ and IL-6 (Agnello et al., 2000).

Another relevant issue raised from our study is the increased susceptibility to variations in IL-10 expression in females. Of relevance is the fact that the same gender effect is observed in the clinical practice, where the increased susceptibility to depression in women in conditions of decrease estrogen secretion (e.g., premenstrual, during the postpartum period and perimenopauselly) is 
well-known (Osterlund et al., 2005). This increased susceptibility has been attributed to fluctuations in estrogen secretion. In fact, evidence derived from experimental and clinical studies demonstrated an important role of (decreased) estrogens in the pathogenesis of depression but also in the production and bioactivity of a variety of cytokines (Nordell et al., 2003; Suuronen et al., 2005). Estrogens depletion increases the levels of pro-inflammatory cytokines (Bismar et al., 1995), and studies using murine microglia cells showed that estrogens are able to increase IL-10 levels (Dimayuga et al., 2005). Accordingly, our results suggest that in animals that cannot express IL10 , the possible protective effects induced by estrogens are lost since IL- $10^{-1-}$ female mice displayed increased signs of depressive-like behavior when compared with male animals. In contrast, the higher levels of IL-10 present in PMT10 animals were probable bolstered by estrogens, potentiating their protective effects and anti-depressive effects in female mice. Moreover, clinical studies showed that estrogens not only trigger antidepressant-like actions (for review, see (Halbreich and Kahn, 2001)) but also improve the therapeutic action of antidepressants (Soares et al., 2001), specially of those acting in the serotoninergic system(Chang and Chang, 1999; Estrada-Camarena et al., 2006; Lu and Bethea, 2002). Besides the influence of estrogens, other sexual steroids, such as testosterone, might also be implicated in the gender difference observed in depressive-like behavior after IL-10 imbalances. In fact some human and animal studies have shown that testosterone increases IL-10 expression (Liva and Voskuhl, 2001; Malkin et al., 2004) and reduces the expression of pro-inflammatory cytokines such as IL-1 $\beta$, IL-6 and TNF- $\alpha$ (D'Agostino et al., 1999). Taken together, these data highlight a possible interaction between sexual steroids and cytokines actions.

The association between anxiety and depressive disorders is well-known, and there are several common factors involved in both conditions (Cameron, 2006; Cameron et al., 2004; Gulley and Nemeroff, 1993). Therefore, the exploration of anxiety-like signs in experimental models influencing depressive-like behavior becomes relevant. Interestingly, data from the open-field test suggested that IL-10 $0^{-/-}$animals display a hyperanxious phenotype both in males and females. However, this phenotype could not be confirmed in the EPM, a more robust test to assess anxiety-like behavior. A possible explanation for these paradoxical findings could be the increased exploratory behavior evinced by $\mathrm{IL}-10^{-/-}$animals. As the EPM is based on the conflict between the innate exploratory behavior and fear of height and exposed environments, it is likely that the increased tendency for exploration in IL- $10^{-/-}$ might be a confounding effect in this behavioral paradigm and blunt the hyperanxious phenotype. In further support of this view are the findings of decreased anxiety evinced by PMT10 mice. Further studies, using other behavioral tests, are needed to better explore the influence of IL-10 levels in anxiety behavior both in basal and under stressful condition (an approach currently under study in our laboratory).

While the influence of IL-10 on anxiety behavior needs further experimental work, the present data rule out any influence of this anti-inflammatory cytokine in reference memory. In fact, the hippocampus-dependent task used to assess spatial reference memory failed to reveal differences between the performance of both genetically modified mice models and their respective wild-type controls. These findings are of relevance, in the sense that they reveal the specificity of the influence of IL-10 levels in affective/mood conditions.

In conclusion, our behavioral data demonstrate that IL10 , an anti-inflammatory cytokine, is an important molecule in the modulation of depressive-like behavior. This finding calls for a reappraisal of the "cytokine hypothesis of depression", in the sense that imbalances of both proor anti-inflammatory cytokines might modulate mood behavior. Furthermore, the present observations might be of relevance in all those conditions (autoimmune, malignant and infectious disease) associated to polymorphisms of the IL-10 family gene clusters in which depression seems to be more prevalent (Nery et al., 2007; van Boxel-Dezaire et al., 1999; Zorzon et al., 2001).

\section{Conflict of interest}

There are no financial or other conflicts of interest.

\section{Contributors}

Mesquita AR, Correia-Neves M and Sousa N designed the study, wrote the protocol, analyzed the data and wrote the first draft of the manuscript; Mesquita AR performed all behavior experiments and Roque $\mathrm{S}$ the ELISA procedures; Pedrosa $\mathrm{J}$ and Palha JA discussed the results and provided very important comments for the final version of the manuscript; Castro $G$ and Vieira $P$ produced the PMT10 animals and also provided important comments for interpretation of results. All authors contributed to and have approved the final manuscript.

\section{Funding}

This study was unfunded.

\section{Acknowledgements}

We acknowledge the Portuguese Foundation for Science and Technology (FCT) for providing a fellowship to Mesquita AR (SFRH/BD/11838/2003). We also thank Prof. Pedro Oliveira for the helpful comments on the statistical issues.

\section{References}

Agnello D, Villa P, Ghezzi P. Increased tumor necrosis factor and interleukin-6 production in the central nervous system of interleukin10-deficient mice. Brain Research 2000;869:241-3. 
Ando T, Dunn AJ. Mouse tumor necrosis factor-alpha increases brain tryptophan concentrations and norepinephrine metabolism while activating the HPA axis in mice. Neuroimmunomodulation 1999;6:319-29.

Araujo DM, Lapchak PA, Collier B, Quirion R. Localization of interleukin-2 immunoreactivity and interleukin-2 receptors in the rat brain: interaction with the cholinergic system. Brain Research 1989;498:257-66.

Bismar H, Diel I, Ziegler R, Pfeilschifter J. Increased cytokine secretion by human bone marrow cells after menopause or discontinuation of estrogen replacement. Journal of Clinical Endocrinology \& Metababolism 1995;80:3351-5.

Bluthe RM, Castanon N, Pousset F, Bristow A, Ball C, Lestage J, et al. Central injection of IL-10 antagonizes the behavioural effects of lipopolysaccharide in rats. Psychoneuroendocrinology 1999;24:301-11.

Boguszewski P, Zagrodzka J. Emotional changes related to age in rats - a behavioral analysis. Behavioural Brain Research 2002;133:323-32.

Brebner K, Hayley S, Zacharko R, Merali Z, Anisman H. Synergistic effects of interleukin-1beta, interleukin-6, and tumor necrosis factoralpha: central monoamine, corticosterone, and behavioral variations. Neuropsychopharmacology 2000;22:566-80.

Breder CD, Dinarello CA, Saper CB. Interleukin-1 immunoreactive innervation of the human hypothalamus. Science 1988;240:321-4.

Cameron OG. Anxious-depressive comorbidity: effects on HPA axis and CNS noradrenergic functions. Essential Psychopharmacology 2006;7:24-34.

Cameron OG, Abelson JL, Young EA. Anxious and depressive disorders and their comorbidity: effect on central nervous system noradrenergic function. Biological Psychiatry 2004;56:875-83.

Capuron L, Ravaud A, Dantzer R. Early depressive symptoms in cancer patients receiving interleukin 2 and/or interferon alfa- $2 b$ therapy. Journal of Clinical Oncology 2000;18:2143-51.

Carroll BJ, Martin FI, Davies B. Pituitary-adrenal function in depression. Lancet 1968;1:1373-4.

Chang AS, Chang SM. Nongenomic steroidal modulation of high-affinity serotonin transport. Biochimica et Biophysica Acta 1999;1417:157-66.

Crane JW, Buller KM, Day TA. Evidence that the bed nucleus of the stria terminalis contributes to the modulation of hypophysiotropic corticotropin-releasing factor cell responses to systemic interleukin-1beta. Journal of Comparative Neurology 2003;467:232-42.

Cunningham Jr ET, De Souza EB. Interleukin 1 receptors in the brain and endocrine tissues. Immunology Today 1993;14:171-6.

D’Agostino P, Milano S, Barbera C, Di Bella G, La Rosa M, Ferlazzo V, et al. Sex hormones modulate inflammatory mediators produced by macrophages. Annals of the New York Academy of Sciences 1999;876:426-9.

De La Garza 2nd R. Endotoxin- or pro-inflammatory cytokine-induced sickness behavior as an animal model of depression: focus on anhedonia. Neuroscience \& Behavioral Reviews 2005;29:761-70.

Dimayuga FO, Reed JL, Carnero GA, Wang C, Dimayuga ER, Dimayuga VM, et al. Estrogen and brain inflammation: effects on microglial expression of MHC, costimulatory molecules and cytokines. Journal of Neuroimmunology 2005;161:123-36.

Dunn AJ, Wang J, Ando T. Effects of cytokines on cerebral neurotransmission. Comparison with the effects of stress. Advances in Experimental Medicine and Biology 1999;461:117-27.

Dunn AJ, Swiergiel AH, de Beaurepaire R. Cytokines as mediators of depression: what can we learn from animal studies? Neuroscience \& Biobehavioral Reviews 2005;29:891-909.

Estrada-Camarena E, Lopez-Rubalcava C, Fernandez-Guasti A. Facilitating antidepressant-like actions of estrogens are mediated by 5 HT1A and estrogen receptors in the rat forced swimming test. Psychoneuroendocrinology 2006;31:905-14.

Fiorentino DF, Zlotnik A, Mosmann TR, Howard M, O'Garra A. IL-10 inhibits cytokine production by activated macrophages. Journal of Immunology 1991;147:3815-22.

Gulley LR, Nemeroff CB. The neurobiological basis of mixed depressionanxiety states. Journal of Clinical Psychiatry 1993;54(Suppl.):16-9.
Halbreich U, Kahn LS. Role of estrogen in the aetiology and treatment of mood disorders. CNS Drugs 2001;15:797-817.

Harvey D, Smith R, English K, Mahon B, Commins S. Interleukin-10 (IL10) but not Lipopolysaccharide (LPS) produces increased motor activity and abnormal exploratory patterns while impairing spatial learning in Balb/c mice. Physiology \& Behavior 2006;87:842-7.

Hayley S, Staines W, Merali Z, Anisman H. Time-dependent sensitization of corticotropin-releasing hormone, arginine vasopressin and c-fos immunoreactivity within the mouse brain in response to tumor necrosis factor-alpha. Neuroscience 2001;106:137-48.

Hayley S, Brebner K, Lacosta S, Merali Z, Anisman H. Sensitization to the effects of tumor necrosis factor-alpha: neuroendocrine, central monoamine, and behavioral variations. Journal of Neuroscience 1999;19:5654-65.

Kubera M, Lin AH, Kenis G, Bosmans E, van Bockstaele D, Maes M. Anti-inflammatory effects of antidepressants through suppression of the interferon-gamma/interleukin-10 production ratio. Journal of Clinical Psychopharmacology 2001;21:199-206.

Lanquillon S, Krieg JC, Bening-Abu-Shach U, Vedder H. Cytokine production and treatment response in major depressive disorder. Neuropsychopharmacology 2000;22:370-9.

Leon LR, Kozak W, Rudolph K, Kluger MJ. An antipyretic role for interleukin-10 in LPS fever in mice. American Journal of Physiology 1999;276:R81-9.

Linthorst AC, Flachskamm C, Muller-Preuss P, Holsboer F, Reul JM. Effect of bacterial endotoxin and interleukin-1 beta on hippocampal serotonergic neurotransmission, behavioral activity, and free corticosterone levels: an in vivo microdialysis study. Journal of Neuroscience 1995; 15:2920-34.

Liva SM, Voskuhl RR. Testosterone acts directly on CD4+ T lymphocytes to increase IL-10 production. Journal of Immunology 2001;167:2060-7.

Lu NZ, Bethea CL. Ovarian steroid regulation of 5-HT1A receptor binding and $\mathrm{G}$ protein activation in female monkeys. Neuropsychopharmacology 2002;27:12-24.

Maes M. Major depression and activation of the inflammatory response system. Advances in Experimental Medicine and Biology 1999;461:25-46

Maes M, Bosmans E, Meltzer HY, Scharpe S, Suy E. Interleukin-1 beta: a putative mediator of HPA axis hyperactivity in major depression? American Journal of Psychiatry 1993;150:1189-93.

Maes M, Bosmans E, Meltzer HY. Immunoendocrine aspects of major depression. Relationships between plasma interleukin-6 and soluble interleukin-2 receptor, prolactin and cortisol. European Archives of Psychiatry and Clinical Neuroscience 1995a;245:172-8.

Maes M, Meltzer HY, Bosmans E, Bergmans R, Vandoolaeghe E, Ranjan $\mathrm{R}$, et al. Increased plasma concentrations of interleukin-6, soluble interleukin-6, soluble interleukin-2 and transferrin receptor in major depression. Journal of Affective Disorders 1995b;34:301-9.

Maes M, Song C, Lin AH, Bonaccorso S, Kenis G, De Jongh R, et al. Negative immunoregulatory effects of antidepressants: inhibition of interferon-gamma and stimulation of interleukin-10 secretion. Neuropsychopharmacology 1999;20:370-9.

Malkin CJ, Pugh PJ, Jones RD, Kapoor D, Channer KS, Jones TH. The effect of testosterone replacement on endogenous inflammatory cytokines and lipid profiles in hypogonadal men. Journal of Clinical Endocrinology \& Metabolism 2004;89:3313-8.

McGeer PL, McGeer EG. The inflammatory response system of brain: implications for therapy of Alzheimer and other neurodegenerative diseases. Brain Research Brain Research Reviews 1995;21:195-218.

Merali Z, Lacosta S, Anisman H. Effects of interleukin-1beta and mild stress on alterations of norepinephrine, dopamine and serotonin neurotransmission: a regional microdialysis study. Brain Research 1997;761:225-35.

Mikova O, Yakimova R, Bosmans E, Kenis G, Maes M. Increased serum tumor necrosis factor alpha concentrations in major depression and multiple sclerosis. European Journal of Neuropsychopharmacology 2001;11:203-8. 
Moore KW, de Waal Malefyt R, Coffman RL, O'Garra A. Interleukin-10 and the interleukin-10 receptor. Annual Review of Immunology 2001;19:683-765.

Musselman DL, Lawson DH, Gumnick JF, Manatunga AK, Penna S, Goodkin RS, et al. Paroxetine for the prevention of depression induced by high-dose interferon alfa. New England Journal of Medicine 2001;344:961-6.

Nava F, Calapai G, Facciola G, Cuzzocrea S, Marciano MC, De Sarro A, et al. Effects of interleukin-10 on water intake, locomotory activity, and rectal temperature in rat treated with endotoxin. International Journal of Immunopharmacology 1997;19:31-8.

Nery FG, Borba EF, Hatch JP, Soares JC, Bonfa E, Neto FL. Major depressive disorder and disease activity in systemic lupus erythematosus. Comprehensive Psychiatry 2007;48:14-9.

Nordell VL, Scarborough MM, Buchanan AK, Sohrabji F. Differential effects of estrogen in the injured forebrain of young adult and reproductive senescent animals. Neurobiology of Aging $2003 ; 24: 733-43$.

Osterlund MK, Witt MR, Gustafsson JA. Estrogen action in mood and neurodegenerative disorders: estrogenic compounds with selective properties - the next generation of therapeutics. Endocrine 2005;28:235-42.

Papanicolaou DA, Wilder RL, Manolagas SC, Chrousos GP. The pathophysiologic roles of interleukin-6 in human disease. Annals of Internal Medicine 1998;128:127-37.

Peterson MG, Mercer JF. Structure and regulation of the sheep metallothionein-Ia gene. European Journal of Biochemistry 1986;160:579-85.

Plotsky PM, Owens MJ, Nemeroff CB. Psychoneuroendocrinology of depression. Hypothalamic-pituitary-adrenal axis. Psychiatric Clinics of North America 1998;21:293-307.

Ramos A, Berton O, Mormede P, Chaouloff F. A multiple-test study of anxiety-related behaviours in six inbred rat strains. Behavioural Brain Research 1997;85:57-69.

Schiepers OJ, Wichers MC, Maes M. Cytokines and major depression. Progress in Neuropsychopharmacology \& Biological Psychiatry 2005;29:201-17.

Shintani F, Kanba S, Nakaki T, Nibuya M, Kinoshita N, Suzuki E, et al. Interleukin-1 beta augments release of norepinephrine, dopamine, and serotonin in the rat anterior hypothalamus. Journal of Neuroscience 1993;13:3574-81.

Silverman MN, Macdougall MG, Hu F, Pace TW, Raison CL, Miller AH. Endogenous glucocorticoids protect against TNF-alpha-induced increases in anxiety-like behavior in virally infected mice. Molecular Psychiatry 2007;12:408-17.
Simen BB, Duman CH, Simen AA, Duman RS. TNFalpha signaling in depression and anxiety: behavioral consequences of individual receptor targeting. Biological Psychiatry 2006;59:775-85.

Sluzewska A, Rybakowski JK, Laciak M, Mackiewicz A, Sobieska M, Wiktorowicz K. Interleukin-6 serum levels in depressed patients before and after treatment with fluoxetine. Annals of the New York Academy of Sciences 1995;762:474-6.

Smith EM, Cadet P, Stefano GB, Opp MR, Hughes Jr TK. IL-10 as a mediator in the HPA axis and brain. Journal of Neuroimmunology 1999; 100:140-8.

Soares CN, Almeida OP, Joffe H, Cohen LS. Efficacy of estradiol for the treatment of depressive disorders in perimenopausal women: a doubleblind, randomized, placebo-controlled trial. Archives of General Psychiatry 2001;58:529-34.

Song C, Merali Z, Anisman H. Variations of nucleus accumbens dopamine and serotonin following systemic interleukin-1, interleukin-2 or interleukin-6 treatment. Neuroscience 1999;88:823-36.

Sousa MM, Fernandes R, Palha JA, Taboada A, Vieira P, Saraiva MJ. Evidence for early cytotoxic aggregates in transgenic mice for human transthyretin Leu55Pro. American Journal of Pathology 2002;161:1935-48.

Suuronen T, Nuutinen T, Huuskonen J, Ojala J, Thornell A, Salminen A. Anti-inflammatory effect of selective estrogen receptor modulators (SERMs) in microglial cells. Inflammation Research 2005;54:194-203.

Tilders FJ, Schmidt ED. Interleukin-1-induced plasticity of hypothalamic CRH neurons and long-term stress hyperresponsiveness. Annals of the New York Academy of Sciences 1998;840:65-73.

Tuglu C, Kara SH, Caliyurt O, Vardar E, Abay E. Increased serum tumor necrosis factor-alpha levels and treatment response in major depressive disorder. Psychopharmacology (Berlin) 2003;170:429-33.

van Boxel-Dezaire AH, Hoff SC, van Oosten BW, Verweij CL, Drager AM, Ader HJ, et al. Decreased interleukin-10 and increased interleukin-12p40 mRNA are associated with disease activity and characterize different disease stages in multiple sclerosis. Annals of Neurology 1999;45:695-703.

Yirmiya R, Pollak Y, Morag M, Reichenberg A, Barak O, Avitsur R, et al. Illness, cytokines, and depression. Annals of the New York Academy of Sciences 2000;917:478-87.

Yirmiya R, Pollak Y, Barak O, Avitsur R, Ovadia H, Bette M, et al. Effects of antidepressant drugs on the behavioral and physiological responses to lipopolysaccharide (LPS) in rodents. Neuropsychopharmacology 2001;24:531-44.

Zorzon M, de Masi R, Nasuelli D, Ukmar M, Mucelli RP, Cazzato G, et al. Depression and anxiety in multiple sclerosis. A clinical and MRI study in 95 subjects. Journal of Neurology 2001;248:416-21. 